\title{
Violación de los derechos humanos y laborales, casos de empresas piñeras en la zona norte, su exposición y denuncia en los campus universitaros estatales
}

\author{
Human and Labor Rights Violations, Cases of Pineapple \\ Farms in the Northern Region of Costa Rica, Their \\ Exposure, and Complaints in Different Public Universities \\ Around the Country
}

\author{
Julio Alfaro Alvarado \\ Estudiante de Contabilidad y Finanzas \\ Universidad Técnica Nacional \\ Sede Central, Alajuela \\ juliode11985@gmail.com \\ Marlon Webb Castillo \\ Estudiante de Gestión Ambiental \\ Universidad Nacional de Costa Rica \\ Sede Central, Campus Omar Dengo \\ marwebb1996@gmail.com
}

Recibido: 13/11/18 Aceptado: 31/01/19

Resumen. El propósito de la presente investigación es mostrar la situación que se ha venido dando en Costa Rica con respecto a la expansión piñera y sus consecuencias en la violación de los derechos humanos básicos y laborales en dicha industria, este como punto principal dentro de nuestra investigación. El estudio se fundamenta en testimonios autorizados tanto de carácter anónimo como manifiesto de colaboradores actuales y pasados, los cuales relatan vivencias dentro y fuera de sus áreas de trabajo, donde exponen cómo sus derechos han sido violentados por años y cómo estas vivencias han quedado impunes ante el aparato institucional del Estado. Se cuenta con testimonios de una mujer embarazada, la cual fue sometida a trabajos de limpieza forzosos; un extrabajador despedido debido a que fue incapacitado por una condición crónica, teniendo solo una semana de trabajar; y trabajadores que han sido perseguidos por las 
mismas empresas, que consideran el ser sindical como un posible elemento rebelde y de oposición. Se menciona también la falta de responsabilidad ambiental por parte de la industria y cómo esto ha afectado de manera negativa al medio ambiente y los derechos humanos. Por otro lado, se investiga la existencia de organizaciones que buscan proteger a los exempleados o personas afectadas por la expansión de esta industria, amparándolas y brindándoles un soporte a nivel emocional. Por último, mencionamos el trabajo desde el movimiento estudiantil universitario y la reflexión que ha generado en torno a esta problemática.

Palabras clave: piñera, derechos humanos, movimiento estudiantil, medio ambiente, protección

\begin{abstract}
The purpose of this investigation is to show the expansion of pineapple farms and the consequences of violating labor and human rights of workers within this industry: it is a widespread situation nowadays in Costa Rica. These are the main points in our research. The study is based on authorized testimonies given anonymously or not by current and past workers; they share their experiences inside and outside their workplaces. They expose how their rights have been violated for years and how these experiences have remained unpunished before the institutional apparatus of the state. A pregnant woman reveals that she was subjected to forced cleaning work; an ex worker tells that he was fired one week after returning from a sickness leave for health reasons due to a chronic health condition. There are also testimonies of workers who have been persecuted by the same companies; they were considered as possible opponents and rebellious elements to the company. It will be mentioned how this industry has a lack of commitment to environmental responsibility and how this has a negative impact on the environment and human rights. Furthermore, the study will consider those organizations seeking to protect former employees or people affected by the expansion of this industry, and how these organizations have managed to protect these people and provide emotional support. Finally, this situation will be studied to show how it has been addressed by the university student movement and the reflection generated.
\end{abstract}

Keywords: pineapple farm, Human Rights, student movement, environment, protection.

\title{
Introducción
}

Como parte de una labor investigativa y accionaria dentro del colectivo ambientalista en el que colaboramos como voluntarios hemos decidido presentar esta ponencia, también por la controversia que se ha venido dando alrededor de las empresas que se dedican al monocultivo de piña (conocidas 
URL: http://www.revistas.una.ac.cr/index.php/dialogo/index

CORREO ELECTRÓNICO: universidadendialogo@una.cr

DOI: https://doi.org/10.15359/udre.9-1.9

como piñeras), debido al malestar de comunidades vecinas y trabajadores de estas empresas que señalan la falta de derechos humanos y laborales, al no apegarse a los lineamientos del Código de Trabajo costarricense ni a la Declaratoria Universal de los Derechos Humanos de la Unesco, hecho que no se denuncia públicamente por miedo a represalias.

Se contará, como parte de esta investigación, con el aporte de testimonios, algunos de carácter anónimo (para proteger la identidad de las personas que aún siguen laborando en estas empresas), así como con testimonios manifiestos de extrabajadores que denuncian malos tratos, despidos por recurrir a los servicios de salud en situaciones urgentes, exposición a agroquímicos, entre otros.

Asimismo, se mencionan acciones vinculadas a la forma en que se ha abordado el tema en los distintos campus universitarios y el impacto que ha tenido en los estudiantes.

\section{Formulación del problema}

Con base en la investigación que se ha desarrollado sobre este tema, los autores han llegado a las siguientes interrogantes: ¿Cuáles han sido los derechos humanos y laborales que se han violado en estas empresas y qué tan importante es la acción de los movimientos estudiantiles y la denuncia pública en campus universitarios de problemáticas como las irregularidades en las empresas piñeras que denuncian personas afectadas?

\section{Justificación}

Esta investigación surge debido a los múltiples contactos de denuncia que se han ido propiciando con personas afectadas, por la falta de regulación o acción del Estado en empresas de monocultivo, en este caso de cultivo desmedido de piña.

Durante el desarrollo de este proceso investigativo se han encontrado documentados alrededor de cinco casos en que las personas afectadas relatan los abusos sobre sus derechos, que se han visto vulnerados y violentados en el momento de ingresar a trabajar en una piñera.

Los testimonios, sumamente valiosos, servirán para evidenciar la problemática que enfrentan estas personas, así como datos obtenidos por grupos ambientalistas, testimonios del impacto de los diferentes conversatorios y foros que se han hecho sobre este tema en las universidades estatales y la reacción de la comunidad estudiantil ante los mismos. 


\section{Reseña histórica sobre la industria piñera}

Según Henry Picado, presidente de la Federación Costarricense para la Conservación del Ambiente,

La experimentación de siembra de piña comenzó con la empresa Dole, de nuevas variedades a partir de los años 60 , su comienzo se dio en la Zona Sur. Alrededor del año de 1984, debido a los problemas de siembra de piña en Hawái (acidificación del suelo y menor humedad), se traslada la producción en masa a nuestro país, en la zona norte desde el año aproximadamente 1984. Se abre el mercado internacional de la piña, a partir de la política de agricultura para el cambio bajo el gobierno de Óscar Arias, se comienzan a dar las nuevas plantaciones con un carácter comercial, así como mayor experimentación de siembra de variedades de piña. (Picado, comunicación personal, 7 de julio del 2017).

A partir del período 2000-2001, se tenían unas 10000 HA de piña sembradas en el sur, de la variedad Golden (por su resistencia al clima más húmedo y los suelos más pantanosos). Posteriormente, debido al auge del negocio y las ganancias que generaba, además de que el Estado comenzó a subsidiar estos proyectos mediante los CAT (créditos de abonos tributarios), se da una expansión creciente exponencial en el período 2003-2005.

Debido a los problemas de violación de los derechos humanos, laborales y ambientales, en el 2003 se da una lucha contra la empresa Pindeco y se crea el Frente de Lucha Contra Pindeco, el cual estableció demandas y luchó en contra de la explotación laboral principalmente.

Comienza la gran expansión del 2005 al 2014, cuantificando unas 37 659,9 HA para este último año (INEC, Censo Agropecuario, 2015). De la misma manera que el Frente de Lucha Contra Pindeco y en conjunto con el Foro Emaus, en el 2007 nace el Frente Nacional de Sectores Afectados por la Producción Piñera (Frenasapp).

De acuerdo con doña Erlinda Quesada, miembro activa del Frenasap p,

Se empieza a generar una cuantificación de daños ambientales en mantos acuíferos, tomas de agua potable para consumo humano, la salubridad de las personas. Debido a las denuncias, se pararon proyectos de piñeras, como el caso de Tico Verde en Guácimo, se denunció la contaminación de nacientes de agua en Milano, El Cairo y la Francia (provincia de 
Limón) para el 2007. Esta empresa tuvo que enfrentar un juicio, que se reanudó en el año 2009. (Quesada, comunicación vía telefónica, 7 de julio del 2017)

Para el 2007, Costa Rica desplaza a Hawái y se convierte en el primer productor de piña en el mundo, mientras Hawái empieza a dedicarse al turismo ecológico. Entre el 2003 y el 2009 se presentaron 120 denuncias contra la industria piñera en materia ambiental, además en el mismo período se da una disminución de indicadores de desarrollo social. Para el 2008, debido a la preocupación de ecologistas, sindicalistas y defensores de la salud pública, el Gobierno intenta reactivar el cultivo de granos básicos, iniciativa que no pudo realizarse, ya que la mayoría de tierras para cultivo están ocupadas por piña, 40000 hectáreas cultivadas donde trabajan unos 20000 peones, en condiciones cuestionables (Boeglin, 2015).

Según Picado, "el sector piñero para el año 2011 presentaba un aumento anual del $8 \%$, lo que podría significar que, así como aumenta el sector piñero, aumentan las personas afectadas por dicha industria y los trabajadores en condiciones cuestionables" (comunicación personal, abril del 2017).

En el 2013, en Finca Once, ubicada en Pavón de Los Chiles, alrededor de treinta trabajadores se organizan y marchan, sin importar las posibles represalias que pueda tomar la empresa en su contra por exigir mejores condiciones laborales, ya que la empresa utiliza contratistas para evadir sus responsabilidades laborales y sociales. Hablando del sector piñero, fue un acto de valentía y coraje por parte de los trabajadores que buscaron hacer valer sus derechos, ya que las represalias por parte de este sector productivo contra sus trabajadores pasan desapercibidas por las autoridades. Como el caso de la empresa Agromonte, que en el 2015 despidió a setenta empleados que no estaban de acuerdo con perder sus garantías laborales al ser contratados por un contratista externo a la empresa.

El mes de marzo del 2015, la Comisión Interamericana de Derechos Humanos realizó una reunión donde escuchó a las comunidades afectadas por el sector piñero en el país. En el año 2016, Oxfam dio a conocer datos de un estudio en donde señala las condiciones inhumanas a las que se enfrentan las personas que se relacionan con estos cultivos, enfermedades como gastritis o un aumento alarmante de personas con cáncer, además de las largas jornadas de diez a doce horas, sin al menos salarios mínimos, y a cualquier intento de organización son despedidos (Dubois et al., 2016). 
Estos datos de la historia de la producción de piña en el país son alarmantes, se nota a lo largo de los años la poca preocupación y la falta de compromiso de las empresas con las comunidades y el medio ambiente, pero además la actitud complaciente de los gobiernos con este sector. Y es aún más preocupante el comienzo de la exportación de piña con china, lo que significa un aumento en todas estas problemáticas que rodean a este sector.

\section{Marco de referencia}

Expansión piñera:

Proceso industrial de producción masiva de piña que se dio en Costa Rica, debido a la migración de producción de piña de Hawái a nuestro país, el cual tuvo el apoyo del primer Gobierno de Óscar Arias, y ha venido contando con el apoyo de los Gobiernos de turno hasta la fecha. Ha sido cuestionado debido a los múltiples daños ambientales dejados por las empresas allegadas a este sector, así como violaciones a los derechos laborales y humanos que han sufrido los trabajadores de las mismas. (Picado, entrevista personal, 7 de julio del 2017)

En cuanto a los derechos humanos, la Declaración Universal de los Derechos Humanos señala lo siguiente:

Como ideal común por el que todos los pueblos y naciones deben esforzarse, a fin de que tanto los individuos como las instituciones, inspirándose constantemente en ella, promuevan, mediante la enseñanza y la educación, el respeto a estos derechos y libertades, y aseguren, por medidas progresivas de carácter nacional e internacional, su reconocimiento y aplicación universales y efectivos, tanto entre los pueblos de los Estados Miembros como entre los de los territorios colocados bajo su jurisdicción (Unesco, 2008, p. 3).

Derecho laboral: De la definición encontrada en el Diccionario jurídico:

Aquel que tiene por finalidad principal la regulación de las relaciones jurídicas entre empresarios y trabajadores, y de unos y otros con el Estado, en lo referente al trabajo subordinado, y en cuanto atañe a los profesionales y a la forma de prestación de servicios, y también en lo relativo a las consecuencias jurídicas mediatas e inmediatas de la actividad laboral. (Cabanellas, 1993, p. 100) 
Explotación laboral: De la definición encontrada en el libro La exclusión social y el Estado del bienestar en España: "Sería la ausencia del trabajo decente, es decir, la presencia del trabajo precario" (Vidal, 2006, p. 347). Según el autor, bajo dicho término quedarían englobadas las siguientes situaciones, que son comparables a las que incluiría el concepto de precariedad:

Inestabilidad laboral: Sobre todo en lo que tiene que ver con contrataciones temporales no voluntarias. Pero también la inestabilidad asociada al sentimiento de incertidumbre sobre el futuro. Hablamos de rotación en el empleo tras la finalización de estos contratos y los sucesivos períodos de paro intercalados y de las facilidades de despido en los contratos indefinidos. Podríamos finalmente incluir los contratos de jornada parcial, en tanto no sean voluntarios y supongan subempleo.

Ausencia de derechos laborales y sociales regulados: Hablamos de trabajo asalariado productivo sumergido como la modalidad de trabajo informal, que no presupone voluntariedad.

Condiciones laborales precarias: Incluye, entre otras, desigualdad de salarios, malas condiciones de salubridad e higiene y de seguridad laboral (siniestralidad laboral) y ausencia del control y/o autonomía del trabajo (Vidal, 2006).

\section{Marco metodológico}

La información para nuestra ponencia se ha obtenido a través de diálogos y testimonios de trabajadores y personas de las universidades que han ayudado en la difusión y el recibimiento de la información. También por medio de datos e investigaciones de organizaciones no gubernamentales y un instrumento para recolección de datos. Se contó con una entrevista semiestructurada que nos sirvió de guía para obtener la información de las personas, sin embargo, debido a la complejidad que algunos sintieron se prescindió de ella; se anexa al final del documento como prueba investigativa. Se decidió utilizar la herramienta de historias de vida para poder relatar más ampliamente los testimonios.

\section{Testimonios}

Michael: Testimonio de extrabajador de piñera ByJimenez, piñera ubicada en Pavón de Los Chiles; el joven nos contacta a través de las redes sociales para pedirnos asesoría, ya que él fue despedido sin responsabilidad patronal, bajo alegato del patrono de que él provocó una riña dentro de la empresa y 
ReVista UNIVERSIDAd EN Diálogo • Vol. 9, N. ${ }^{\circ}$ 1, Enero-Junio, 2019 • 141-157

ISSN 2215-2849 • EISSN: 2215-4752

URL: http://www.revistas.una.ac.cr/index.php/dialogo/index CORREO ELECTRÓNICO: universidadendialogo@una.cr

DOI: https://doi.org/10.15359/udre.9-1.9

esto generó la baja de rendimiento del personal en el área de planta. Se logró contactar al joven Michael vía telefónica, el cual a través de conversaciones nos fue relatando cómo fue víctima de abusos verbales y físicos por hacer valer sus derechos laborales y los de sus compañeros: horas extra con paga justa, disminución de la jornada laboral a la establecida por el Código de Trabajo, cobertura del médico del Instituto Nacional de Seguros en caso de accidentes, sin sufrir amenazas de despido inmediato, condiciones laborales justas sin violar los derechos humanos.

El joven nos comentó que inclusive dentro de la empresa estaban obligados a trabajar turnos de hasta veintidós horas diarias para poder estar al día con la producción, por un pago por debajo del salario mínimo por ley. Todo esto fue provocando un descontento de su jefe inmediato hacia él, por lo que en una discusión acalorada, después de casi cuatro años de trabajo, fue golpeado brutalmente, con testigos que no pudieron dar una declaración oportuna por miedo a perder su empleo. La golpiza le ocasionó un daño permanente en uno de los hombros.

Gracias a los esfuerzos del colectivo ambientalista, el joven Michael pudo recibir asesoría directa de Nancy Marín, actual viceministra en la parte de Relaciones Laborales del Ministerio de Trabajo y Seguridad Social, que lo refirió a la sede de Alajuela para llegar a un arreglo conciliatorio entre él y su patrono. Además, Michael accedió a dar su testimonio en los campus universitarios de la Universidad Nacional y la Universidad de Costa Rica, el cual fue recibido por universitarios que se solidarizaron y lo apoyaron hasta con ayuda financiera y lo motivaron a continuar con sus estudios de secundaria. Actualmente, Michael sigue estudiando, no logra encontrar empleo en la zona debido a que los dueños de las piñeras corrieron la voz de su acción y no le dan empleo y se le está apoyando para que concrete una demanda laboral contra la empresa que lo despidió tan abruptamente.

Juan Carlos: Ciudadano costarricense, oriundo de Colonia, Puntarenas, en Los Chiles. Él nos contactó vía web, a través de la página del Bloque Verde, mediante la plataforma de mensajes. Nos cuenta que fue contratado para laborar con manipulación de químicos en una bodega de la empresa Upala Agrícola. Cuando ingresó a trabajar el 22 de mayo del presente año informó a la profesional de la salud sobre un padecimiento crónico que él tiene y alertó también a la persona de Recursos Humanos sobre el peligro de su exposición y posible afectación de salud. Las personas le dijeron que no había problema y que hiciera el intento. Él solicitó ser trasladado al área de trabajo de campo, pero no le dieron la opción. Nueve días después de ingresar a trabajar en la 
bodega de químicos, cayó enfermo y tuvo que acudir al Instituto Nacional de Seguros para recibir asistencia médica, por lo que fue incapacitado por varios días. Cuando volvió a su lugar de trabajo, fue despedido de manera abrupta sin derecho a ningún pago. En este caso, se evidencia una clara violación a los derechos laborales y humanos, tomando en cuenta que don Juan Carlos contaba con una condición médica que le podría afectar debido a la exposición constante con químicos y no contar con el equipo especial necesario para manipular los mismos sin afectar su salud.

Testimonio anónimo: Trabajadora de una empacadora de piña, ella nos contacta por la página web oficial del Bloque Verde en Facebook, logramos que a través de ese medio nos de su número de teléfono. Una vez contactada vía telefónica, nos comenta que ella sufre constante acoso y explotación laboral, debido a que es obligada a hacer trabajo forzado, a sabiendas de su condición de mujer embarazada. Su jefe inmediato la amenaza y le dice que la puede despedir por bajo rendimiento, al parecer su jefe desconocía la legislación laboral vigente de protección a la mujer en estado de gravidez. Ella se encontraba bastante angustiada y tuvo varias discusiones con su jefe. Gracias al apoyo de un profesional en derecho ambiental y otro experto en materia de derechos laborales, se le pudo dar asesoría sin costo, incluyendo afiches del Ministerio de Trabajo relacionados con los derechos y deberes de las mujeres embarazadas y las obligaciones que el patrono posee para con esta población. Una vez asesorada y después de una incapacidad, dada por el médico de la Caja Costarricense del Seguro Social, logró tener una sesión con su jefe y le advirtió de las obligaciones que el patrono tenía con ella, por lo que desde ese instante él mismo decidió no acosarla más, alegando no querer buscarse problemas con órganos judiciales.

Testimonio anónimo, masculino, alrededor de 47 años, trabajador de una piñera, sindicalista: Nos contacta a través de nuestro sitio web para comentarnos que en la empresa en la que labora, Agrícola Agromonte, los trabajadores tomaban su tiempo de desayuno al aire libre expuestos a químicos dañinos y les daban un mínimo tiempo de veinte minutos para poder tener su receso (se adjuntan fotos), además lavaban los tanques y tiraban el agua cerca de nacientes, creando contaminación. El mismo señor nos detalló el estimado de lo que ganaban como peones en piñeras y que no corresponde ni al salario mínimo. Cuando el video y las fotos se hicieron públicas a través de las redes sociales, al compañero y su equipo de trabajo les hicieron amenazas y les prohibieron el uso del teléfono móvil dentro del área de trabajo. Actualmente, debido a la situación adversa y la escasez de empleo en donde él reside, sigue trabajando en la piñera. 


\section{Situación actual}

La situación actual sigue en tensión, comunidades molestas, empresas irresponsables yun Gobierno complaciente. El cultivo depiña sigue en expansión y empresas existentes continúan con irregularidades socioambientales que no son denunciadas por las comunidades afectadas por temor a represalias. Casos como la venta de la finca FINMAC, alrededor de 240 hectáreas, en Pueblo Nuevo, Guácimo, Limón. Finca de cacao orgánico y hogar de perezosos que se empezará a convertir pronto en un enclave piñero, es un ejemplo de esto. Lo que da como resultado más comunidades envueltas en estas problemáticas, sin las regulaciones necesarias y empresas con el apoyo del Gobierno, ya que, según este, es la respuesta al desempleo, empleo a un costo socioambiental muy alto y en el cual los trabajadores se enfrentan a condiciones difíciles.

En los campus universitarios estatales, los movimientos estudiantiles han logrado apertura al tema y su difusión, se ha mantenido el tema de las problemáticas socioambientales en diferentes espacios, permitiendo que el tema no muera y siga generando acciones desde el movimiento estudiantil. Tal es el caso de Michael, traído gracias al apoyo financiero de la Federación Ecologista Costarricense, el cual estuvo en los campus universitarios de la Universidad Nacional y la Universidad de Costa Rica, en espacios seguros, denunciando los abusos sufridos por la empresa en la que trabajó casi cuatro años. Diversos debates se han dado, tal como el caso del debate entre el empresario piñero Jorge Volio y la activista y socióloga Eva Carazo, para el caso de la Universidad Técnica Nacional, en su sede de San Carlos. El Instituto Tecnológico de Costa Rica en su sede de Cartago ha hecho recientemente un debate sobre el tema de las piñeras, generando diferentes reacciones sobre el tema. Además, en la Universidad Nacional, sede Omar Dengo, continúan realizándose conversatorios con personas afectadas por la expansión piñera en la Zona Norte, como en el caso de la "Semana de Ciencias Sociales 2017", a la cual se invitaron dos ganaderos afectados por la mosca de la piña y en la "Semana Ambiental 2017" se invita a CANAPEP a un conversatorio para que respondiera dudas de los estudiantes y escuchar su contraparte.

También, se están empezando a realizar reuniones y mesas de diálogo desde el movimiento estudiantil de la Universidad Nacional para acompañar a comunidades a las cuales la expansión piñera está llegando o comunidades que ya se están viendo afectadas por empresas piñeras. 
URL: http://www.revistas.una.ac.cr/index.php/dialogo/index

CORREO ELECTRÓNICO: universidadendialogo@una.cr

DOI: https://doi.org/10.15359/udre.9-1.9

Desde las diferentes universidades públicas, asociaciones estudiantiles y movimientos autónomos como los Frentes Ecologistas Universitarios, siguen realizando distintas actividades con respecto al tema, con el objetivo de generar conciencia en los estudiantes y tomar acciones cada vez más vinculantes, sin embargo, aunque las acciones continúan, se han ido reduciendo en los últimos meses.

\section{Difusión en los campus universitarios y reacciones}

El tema ha generado diferentes reacciones dentro de las universidades estatales, desde posturas a favor, defendiendo la generación de empleos directos e indirectos, ataque a los grupos ecologistas, alegando el hecho de que no proponen soluciones alternas para reducir el desempleo que tenemos en el país, que ronda alrededor del 9.1\%, según el Instituto Nacional de Estadísticas y Censos, léase INEC, en su encuesta del 2017; así como también ha construido posturas en contra, debido a la violaciones de derechos laborales y humanos, condiciones de trabajo insalubres, malos tratos por parte de los patronos e inclusive crisis para otros sectores como el sector papayero y el sector ganadero (INEC, 2017). Los movimientos estudiantiles han tomado posturas, en su mayoría, contra la expansión piñera, pidiendo un alto hasta que no se normalicen las condiciones laborales para los trabajadores, un alto a las violaciones de derechos humanos básicos como el acceso al agua potable, un alto a la contaminación de nacientes, erosión de suelos, un alto al uso excesivo de agrovenenos que desembocan en tratamientos muy costosos para nuestros sistemas de salud pública, como los tratamientos por cáncer, hipertensión, gastritis crónicas y fatiga extrema.

El tema de la moratoria a la expansión piñera en el movimiento estudiantil de las universidades públicas empieza con fuerza a finales de abril del presente año, cuando organizaciones ambientalistas hacen un llamado a las calles para el día 15 de mayo hacia la asamblea legislativa, en defensa del humedal Térraba-Sierpe. Marcha que daría inicio a diferentes acciones en las universidades. Estudiantes ya conscientes de la problemática acuerpan el llamado y empiezan a actuar.

Desde las diferentes universidades públicas empiezan a organizarse actividades desde la comunidad estudiantil a partir de mayo, no solo las típicas mantas que han caracterizado a los movimientos estudiantiles para asistir a la marcha en defensa del humedal Térraba-Sierpe, sino que además se empiezan a organizar cineforos, que buscan informar a la población estudiantil antes de la marcha. 
Según Picado,

Varias marchas se generan en distintas partes del país, acuerpadas por la población estudiantil: Los Chiles, 2 de mayo. Osa, 15 de junio. Siquirres, 19 de junio. También, a raíz de investigaciones de organismos internacionales, como la OXFAM, se logra una campaña contra el consumo de piña costarricense en Europa, fuera de los supermercados LIDL en Alemania. (entrevista personal, 7 de julio del 2017).

Las marchas logran generar presión para la prohibición del bromacil en el país, por medio de un decreto del MAG y se cierran tres piñeras por parte de Setena, en la Zona Norte. Además, empiezan a organizarse movimientos estudiantiles ambientalistas en las universidades públicas en las que no los había, como la Universidad Nacional que, a diferencia de la Universidad de Costa Rica, ha tenido poca acción en estos temas.

El movimiento de lucha por la moratoria a la expansión piñera, que buscaba evitar al menos la expansión en Osa, Zona Sur, y además evidenciar otras problemáticas a nivel nacional relacionadas con las empresas piñeras, tuvo un gran impulso gracias a don Jorge Castro y su esposa Mariana Paniagua, desde Los Chiles, San Carlos, ya que emprenden una caminata de $180 \mathrm{~km}$ hacia Casa Presidencial en el mes de mayo, caminata que genera un gran movimiento en la población estudiantil de las universidades públicas, pero que además daría coraje a trabajadores afectados en las empresas piñeras de la Zona Norte, los cuales estaban viéndose afectados por la violación de sus derechos humanos y laborales y de los cuales exponemos en esta investigación.

Las denuncias de trabajadores y trabajadoras que empezaron a llegar por redes sociales, reuniones y otros medios generaron que se diera la oportunidad de organizar espacios donde estas personas afectadas por la expansión piñera pudieran compartir con estudiantes de las distintas universidades, con el objetivo de concientizar de la importancia de la moratoria a la expansión piñera y la contextualización de la problemática por medio de personas que la viven.

La visita de Michael fue impactante para los estudiantes, ya que se sintieron identificados con un muchacho no muy lejano a su edad relatando los muchos actos de violencia en su contra solo por el hecho de exigir condiciones laborales justas, mínimas y necesarias para el buen desempeño básico de los trabajadores en la empresa en la que laboró. Poder compartir en un espacio con Michael ayudó a los estudiantes a reflexionar sobre las situaciones que viven costarricenses en distintas partes del país y que la problemática es real y no tan lejana. 
URL: http://www.revistas.una.ac.cr/index.php/dialogo/index

CORREO ELECTRÓNICO: universidadendialogo@una.cr

DOI: https://doi.org/10.15359/udre.9-1.9

Además de conversatorios con personas afectadas, se organizan otros espacios como cine foros y debates, por parte del movimiento estudiantil y ambientalista, lo que ha generado una recepción amplia. Mucha de la población estudiantil dentro del sistema universitario estatal tiene al menos una noción de lo que es la expansión piñera, muestran posturas y argumentan las mismas.

\section{Conclusiones y recomendaciones}

Al terminar nuestra investigación y gracias al conocimiento que hemos adquirido llegado a concluir lo siguiente:

- Las grandes corporaciones piñeras buscan obtener ganancias y su único beneficio, sin tomar en cuenta los efectos negativos que generan en las comunidades vecinas a los cultivos de piña como deterioro al ambiente, a la salud y a los recursos naturales.

- Las piñeras tienen condiciones laborales inhumanas e insalubres, a las cuales los trabajadores se ven sometidos en ocasiones por salarios que no llegan al mínimo requerido por el Ministerio de Trabajo y Seguridad Social.

- Los trabajadores que se unen en organizaciones sindicalistas sufren persecución laboral, lo que no les permite tener un respaldo en su trabajo y genera que los patronos se puedan aprovechar obligándolos aceptar condiciones no recomendadas.

- El Estado es complaciente con este sector productivo, ya que deja pasar las irregularidades e históricamente los Gobiernos de turno han tomado pocas medidas para regular este sector.

- La presión social obtiene resultados, más cuando se utilicen herramientas vinculantes como en el caso de la prohibición del Bromacil.

- La comunidad estudiantil universitaria estatal se ha organizado y se ha mantenido activa en el tema, desplegando acciones en contra de las irregularidades socioambientales presentes en las empresas piñeras.

De recomendaciones, se consideran las siguientes:

- Instar a la comunidad estudiantil universitaria a unificarse para tomar acciones en conjunto, vinculantes con el Estado y las comunidades. También informarse más sobre el tema de la industria del monocultivo de piña y sus impactos, organizarse en grupos como los Frentes Ecologistas, que han surgido por esta problemática y han encabezado la lucha por la moratoria a la expansión piñera. 
- Investigar más a fondo otros casos de violaciones de derechos humanos y laborales dentro de las empresas dedicadas a este sector en el país., que permita seguir conociendo casos con los que podamos contextualizar mejor la situación y denunciar.

- Al Estado tomar medidas legislativas y económicas vinculantes que permitan regular al sector piñero y los impactos negativos que este tiene, además de cumplir con la moratoria a la expansión piñera prometida en campaña.

- A la sociedad en general, presionar a estas empresas para que realicen las cosas bien y además que soliciten al Estado la moratoria a la expansión piñera hasta que las empresas y cultivos ya presentes cumplan con toda la legislación nacional e internacional.

- Comprometerse con el pueblo de Costa Rica y hacer las cosas bien son los únicos beneficiarios de esta producción explotadora, destructiva, invasiva y poco sostenible.

\section{Referencias bibliográficas}

Boeglin, N. (2015). La piña de Costa Rica ante la Comisión Interamericana de Derechos Humanos. Diario Digital El País. Recuperado de http:// www.elpais.cr/2015/03/18/la-pina-de-costa-rica-ante-la-comisioninteramericana-de-derechos-humanos/

Cabanellas de Torres, G. (1993). Diccionario jurídico elemental (13 ${ }^{\mathrm{a}}$ ed.). Buenos Aires, Argentina: Editorial Heliasta.

Dubois, V. y otros. (2016). Condiciones de producción, impactos humanos $y$ ambientales en el sector piña en Costa Rica. San José, Costa Rica: OXFAM. Recuperado de https://www.oxfam.de/system/files/condiciones laborales_y_ambientales_de_la_pina_en_costa_rica_-_mayo_2016.pdf

Instituto Nacional de Estadística y Censos (2015). VI Censo Nacional Agropecuario. San José, Costa Rica: INEC.

Instituto Nacional de Estadística y Censos (2017). Encuesta continua de empleo al primer trimestre de 2017. Recuperado de http://www.inec.go.cr/sites/ default/files/documetos-biblioteca-virtual/reece-i-t-2017.pdf

Picado, H. (2017). Conversación vía telefónica con Julio Alfaro. San José, Costa Rica. 
URL: http://www.revistas.una.ac.cr/index.php/dialogo/index

CORREO ELECTRÓNICO: universidadendialogo@una.cr

DOI: https://doi.org/10.15359/udre.9-1.9

Quesada, E. (2017). Conversación telefónica con Julio Alfaro. San José, Costa Rica.

Vidal, F. (2006). La exclusión social y el Estado de bienestar en España (V Informe Euhem de politicas sociales). Madrid, España: Editorial Icaria.

Unesco (2008). Declaratoria Universal de los Derechos Humanos. Santiago, Chile.

\section{Anexos}

\section{Anexo 2. Herramienta de cuestionario}

\section{Entrevista para personas afectadas por la expansión piñera en la Zona Norte del país}

El propósito de este cuestionario es recolectar datos de las personas que nos sirvieron de testigos debido a la problemática de la expansión piñera. Las entrevistas serán grabadas solamente para fines de investigación, respetando la confidencialidad de los participantes anónimos, en caso necesario.

1. Nombre:

2. Edad:

3. Género: $\mathrm{M}(\mathrm{)}) \mathrm{F}(\mathrm{)}$

4. Nivel de escolaridad concluido:

Primaria incompleta / Primaria completa / Secundaria incompleta / Secundaria completa / Universitaria incompleta / Universitaria completa / Postgrado

5. Nacionalidad: ( ) Costarricense ( ) Nicaragüense ( ) Otro

6. ¿Actualmente trabaja usted para alguna empresa relacionada con el monocultivo de piña?

Sí ( ) No ( ).

En caso positivo, ¿cuál sería? 
7. ¿Se encuentra usted actualmente sindicalizado? Sí ( ) No ( ).

8. En pocas palabras, ¿qué opina de las empresas de piña en el país?

9. ¿Cree usted que este tipo de empresas ha traído progreso económico para el país?

Sí ( ) No ( )

10. ¿Ha sufrido de algún episodio de represalias dentro de la empresa en la que labora?

Sí ( ) No ( )

11. En caso positivo, podría relatarnos con sus palabras, qué le ha sucedido y el motivo por el cual ha sufrido represalias:

12. ¿Cómo calificaría las condiciones higiénicas dentro del área de trabajo en las piñeras?

Malo ( ) Regular ( ) Bueno ( ) Muy bueno ( )

13. ¿Cómo calificaría las condiciones de salud del área de trabajo en las piñeras?

Malo ( ) Regular ( ) Bueno ( ) Muy bueno ( )

14. ¿Cómo calificaría usted el pago remunerado de obreros o cualquier otro puesto dentro de las empresas de piña en general?

Malo ( ) Regular ( ) Bueno ( ) Muy bueno ( ) 
URL: http://www.revistas.una.ac.cr/index.php/dialogo/index

CORREO ELECTRÓNICO: universidadendialogo@una.cr

DOI: https://doi.org/10.15359/udre.9-1.9

15. ¿Cómo calificaría usted la jornada laboral dentro de las empresas de piña en general?

Mala ( ) Regular ( ) Buena ( ) Muy buena ( )

16. ¿Cómo calificaría usted las condiciones laborales dentro de las empresas de piña en general?

Mala ( ) Regular ( ) Buena ( ) Muy buena ( )

17. ¿Cómo calificaría la labor de su jefe inmediato dentro de las instalaciones de las piñeras?

Mala ( ) Regular ( ) Buena ( ) Muy buena ( )

18. ¿En general, cómo calificaría el trabajo en las instalaciones de las piñeras?

Malo ( ) Regular ( ) Bueno ( ) Muy bueno ( ) 